\title{
Avvento della strumentazione vertebrale nel trattamento delle fratture dorso-Iombari
}

D.G.Marchesi

Clinique Bois-Cerf, Lausanne (CH)

ABSTRACT The introduction of spinal instrumentation in the manage-
ment of thoracolumbar fractures
Fractures of the thoracolumbar spine have been treated conservatively
until the half of the 20th century. Spinal instrumentations (Harrington,
Luque) were initially developed for deformities and only later found appli-
cations in traumatology. The introduction by Roy-Camille of the pedicle
screw fixation as a stronger vertebral anchorage and the development by
Dick of the "internal fixator" for thoracolumbar spinal fractures have com-
pletely changed the methodology for modern management of vertebral
injuries. New fracture classifications and a better understanding of the bio-
mechanics and the pathomorphology of thoracolumbar injuries have led in
the last decade to a better application of spinal instrumentations and the
development of algorithms for selection of the most appropriate fixation not
only to avoid inappropriate long spinal fusion but also to avoid failures and
complications.
scorso le fratture dorso-lombari
Fino a circa la metà del secolo sono sempre state curate in modo ortopedico, combinando un periodo più o meno lungo a letto con una successiva immobilizzazione gessata. I principi del trattamento seguivano quelli esposti da Böhler (Fig. 1), con risultati spesso abbastanza buoni specialmente per le lesioni di tipo osseo, senza instabilità o deformazioni sagittali eccessive e senza deficit neurologici [1-3].

A partire dagli anni Sessanta si sviluppano le prime strumentazioni vertebrali, indirizzate dapprima al trattamento delle deformità e progressivamente anche alla traumatologia dorso-lombare. Il traguardo che si richiedeva alla chirurgia era una mobilizzazione più precoce dei pazienti, al fine di facilitare le cure personali, ridurre la degenza ospedaliera, favorire un reinserimento più rapido nel mondo del lavoro e diminuire i dolori cronici, roppo spesso associati a fratture mal ridotte o mal consolidate. Rispetto ai gessi, le fissazioni di Harrington e Luque, basate su uncini o fili laminari, permettevano una stabilizzazione interna immediata e una migliore correzione, con lo svantaggio però di dover sacrificare tre segmenti vertebrali a ogni lato della frattura e di essere difficilmente applicabili nelle fratture lombari basse e del giunto lombosacrale $[4,5]$.

Una svolta decisiva si è avuta nel 1968 con l'introduzione delle viti peduncolari dovuta a Roy-Camille [6]. Esse permettevano una presa più salda nelle vertebre e quin-

di una migliore riduzione della frattura e una maggiore stabilità del montaggio. La strumentazione che si basava su placche e viti senza stabilità intrinseca tra di esse imponeva ancora di sacrificare due segmenti vertebrali a ogni lato della frattura per offrire una appropriata robustezza al sistema. Si dovrà aspettare il 1977 (fissatore esterno di Magerl) e soprattutto il 1982 (fissatore interno di Dick) per disporre finalmente di un mezzo di fissazione in cui la stabilità tra viti e barre fosse sufficiente per consentire un ancoraggio unicamente nelle vertebre immediatamente adiacenti alla frattura $[7,8]$. Basati su viti peduncolari a stelo lungo (viti di Schanz) saldamente fissate alle due barre con morsetti mobili, questi sistem permettono di manipolare le fratture vertebrali in ogni direzione e soprattutto di effettuare una ligamentotassi per ridurre indirettamente i frammenti ossei compressivi presenti nel canale (Figg. 2,3) Con l'avvento di queste nuove e convincenti strumentazioni e la progressiva diffusione del loro uso in tutto il mondo, è diventato possibile effettuare anche uno studio accurato dei loro risultati [9-11]. Grazie all'introduzione di nuove classificazioni delle fratture dorsolombari, si è potuto così evidenziare che alcune forme di fratture da scoppio erano accompagnate da perdite di correzione o da rotture delle viti se stabilizzate unicamente con una strumentazione peduncolare posteriore [12-14] Queste complicanze dovute a un supporto biomeccanico insuffi-

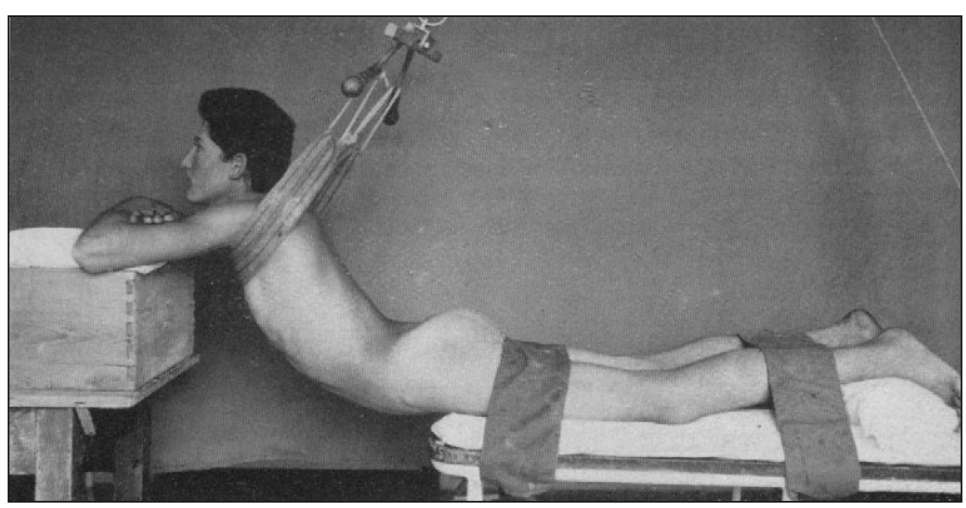

Fig. 1. Tecnica di riduzione delle fratture prima dell'applicazione del bustino gessato (Immagine tratta da: Böhler L, Technique du traitement des fractures, Masson, Paris, 1934)
D01 10.1007/s10261-011-0015-5

ciente della colonna anteriore e talune indicazioni che richiedevano una migliore decompressione delle strutture nervose lese hanno portato allo sviluppo di mezzi di stabilizzazione anteriori, come gabbie e placche, che si sono aggiunti, eventualmente in un secondo tempo operatorio, alla fissazione posteriore [15].

Negli ultimi anni poco è cambiato nella strumentazione vera e propria delle fratture dorso-lombar gravi. Il miglioramento delle tecniche radiologiche, e quindi la migliore valutazione morfologica biomeccanica delle lesioni, ha de terminato la diffusione di nuovial goritmi di trattamento. In alcuni casi si è così riusciti a limitare ul teriormente le aree di artrodesi e

introdurre nuove tecniche chirurgiche meno invasive $[16,17]$. Nei casi meno severi si sono sviluppati metodi di ricostruzione del corpo vertebrale per mezzo di interventi di vertebroplastica, eventualmente combinati con stabilizzazioni peduncolari temporanee talvolta applicate per via percutanea. Queste soluzioni appaiono molto attraenti perché meno invasive di quelle tradizionali, ma non son ancora disponibili dati sufficienti a permetterne la valutazione e la sistematizzazione.

\section{Bibliografia}

Böhler L (1951) Die Technik der Knochenbruchbehandlung. Maudrich Verlag, Vienna

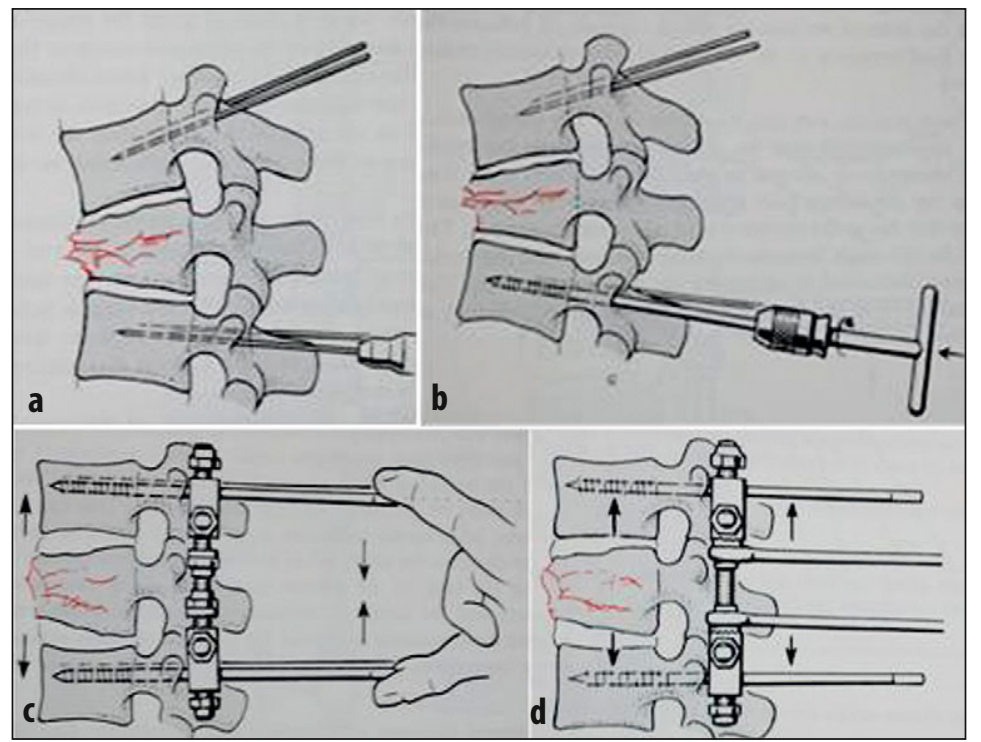

Fig. 2. a-d Applicazione del fissatore interno di Dick per ridurre e stabilizzare una frattura
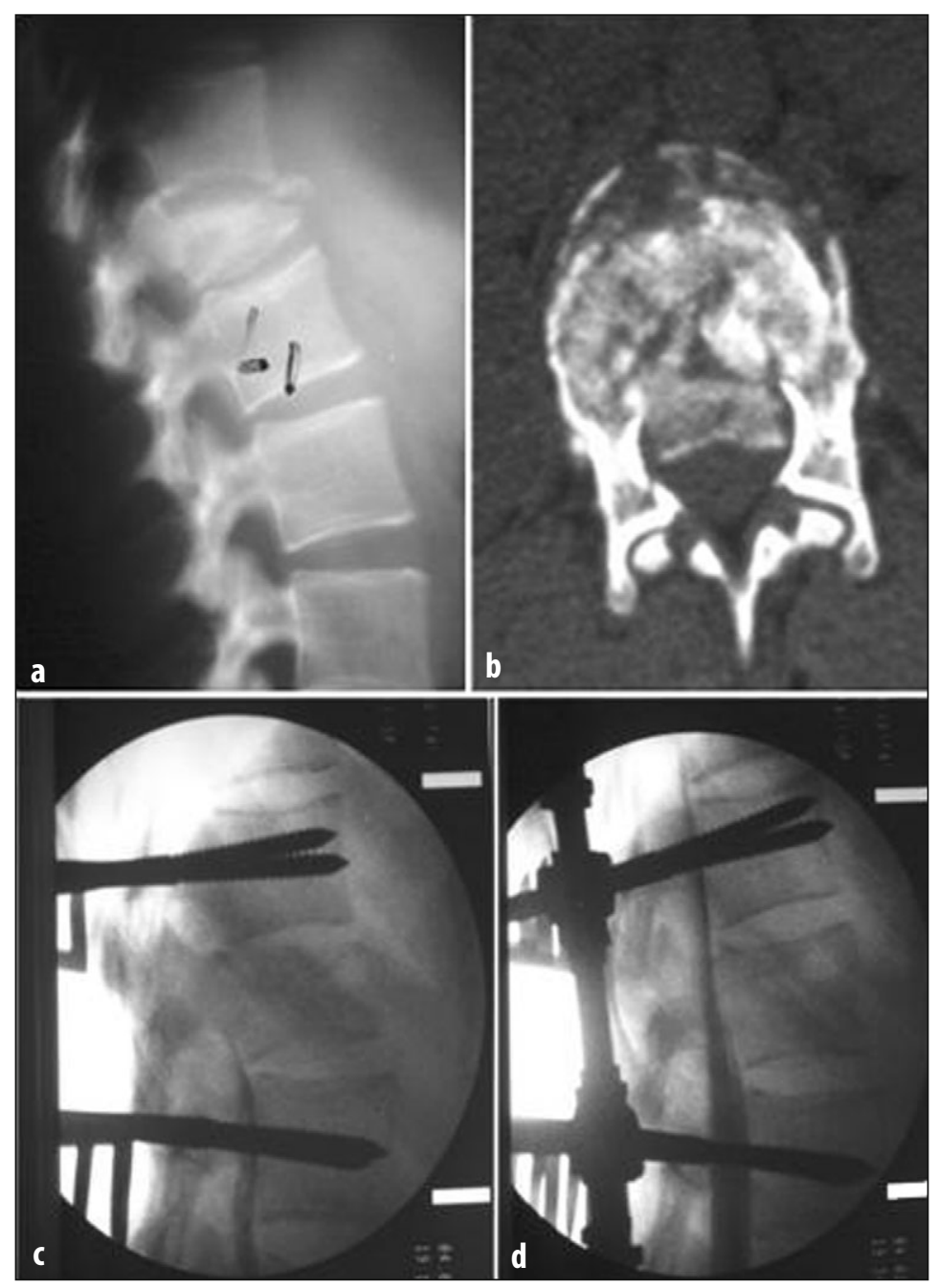
del corpo vertebrale e di liberare indirettamente il canale grazie alla ligamentotassi

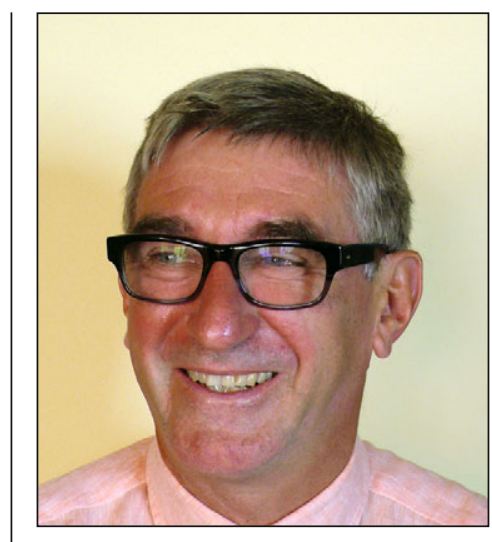

D. G. Marchesi

Mumford J, Weinstein JN, Spratt KF, Goel VK (1993) Thoracolumbar burst fractures. The clinical efficacy and outcome of nonoperative management. Spine 18:955-

Weinstein J, Collalto P, Lehmann TR(1988) Thoracolumbar "burst" fractures treated conservatively: a long-term follow-up. Spine $13 \cdot 33-38$

Harrington PR (1962) Treatment of scoliosis. Correction and internal fixation by spine instrumentation. J Bone Joint Surg Am 44:591610

Luque $\mathrm{E}$ (1982) The anatomic basis and development of segmental spinal instrumentation. Spine 7:256-259

Roy-Camille R, Roy-Camille M, Demeulenaere C (1970) and lumbosacral spine with metallic plates screwed into vertebral pedicles and articular apophyses. Presse Med 78:1447-1448

Dick W, Kluger P, Magerl F et al (1985) A new device for internal fixation of thoracolumbar and lumbar spine fractures: the "fixateur interne". Paraplegia 23:225-

Magerl F (1982) External skeletal fixation of the lower thoracic and upper lumbar spine: current concepts of external fixation of fractures. Springer Verlag, Berlin

Aebi M, Etter C, Kehl T, Thalgott J (1987) Stabilization of the lower thoracic and lumbar spine with the internal spinal skeletal fixation system. Indications, techniques, and first results of treatment. Spine 12:544-551

Dick W (1987) The "fixateur interne" as a versatile implant for spine surgery. Spine 12:882-900 Lindsey RW, Dick W (1991) The fixateur interne in the reduction and stabilization of thoracolumbar spine fractures in patients with neurologic deficit. Spine 16[3 Suppl.]:140-145

Magerl F, Aebi M, Gertzbein SD et al (1994) A comprehensive classification of thoracic and lumbar injuries. Eur Spine J 3:184-201

McCormack, Karaikovic E, Gaines RW (1994) The load sharing classification of spine fractures. Spine 19:1741-1744

McLain RF, Sparling E, Benson DR (1993) Early failure of short-segment pedicle instrumentation for thoracolumbar fractures. A preliminary report. J Bone Joint Surg Am 75:162-16

Kaneda K, Taneichi H, Abumi Ket al (1997) Anterior decompression and stabilization with the Kaneda device for thoracolumbar burs fractures associated with neurological deficits. J Bone Joint Surg Am 79:69-83

Beisse R (2006) Endoscopic surgery on the thoracolumbar junc tion of the spine. Eur Spine J 15:687-704

(2005) Fractures of thoracolumbar spine: monosegmental fixation. Injury 36[Suppl. 2]:90-97 